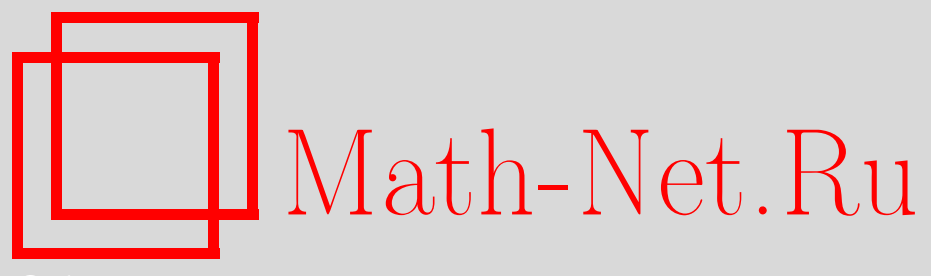

А. В. Беляев, Об асимптотике особых точек решений задачи о движении тяжелого $n$-мерного тела в случае Лагранжа, Матем. сб., 2011, том 202, номер 11, 55-74

DOI: https://doi.org/10.4213/sm7733

Использование Общероссийского математического портала Math-Net.Ru подразумевает, что вы прочитали и согласны с пользовательским соглашением http://www . mathnet.ru/rus/agreement

Параметры загрузки:

IP: 54.197 .217 .227

26 апреля 2023 г., 03:58:37

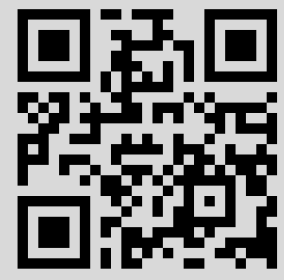




\title{
А. В. Беляев \\ Об асимптотике особых точек решений задачи о движении тяжелого $n$-мерного тела в случае Лагранжа
}

\author{
Доказана однозначность решений задачи о движении тяжелого $n$-мер- \\ ного тела в случае Лагранжа. Найдены все целые решения. \\ Библиография: 23 названия.
}

Ключевые слова: многомерное тело, случай Лагранжа, полная интегрируемость, асимптотика решений, особые точки решений.

\section{§1. Введение}

Доказательство полной интегрируемости многомерного обобщения случая Эйлера задачи о движении твердого тела, полученное Манаковым в [1] методом LA-пары со спектральным параметром, показало весьма перспективную возможность нового взгляда на интегрируемость классических задач механики. Вслед за работой Мищенко и Фоменко [2] в работах Адлера и Мербеке [3], [4] была представлена алгебро-геометрическая схема интегрирования, включающая в себя построение как инволютивного набора первых интегралов, так и LA-пары с параметром. Практически все последующие работы представляли собой иллюстрацию этого метода.

Нельзя не отдать должное красоте конструкции Адлера и Мербеке, однако если акцентировать внимание не на алгебро-геометрической интерпретации фазового пространства, а на более прозаической, но в то же время весьма принципиальной вещи - самих решениях задачи, то эффективность этой конструкции не выглядит впечатляюще.

Так, например, решение классической задачи Лагранжа методом LA-пары в статье Ратиу и Мербеке [5] занимает 20 страниц, в то время как классическое решение занимает в [6] три страницы. Кроме того, пока не существует общего способа получения LA-пары, а если это и удается сделать (см. [7]), то метод не обязательно дает все решения задачи, как это видно на примере задачи Гесса в работе Драговича и Гаджича [8].

Из вышеизложенного следует, что формулировка "задача интегрируется методом LA-пары с параметром", как правило, означает принципиальную возможность получения точных решений и не обязательно означает, что в рассматриваемой задаче получены точные формулы, выражающие явную зависимость искомых величин от времени. Поэтому для проинтегрированной таким способом задачи, вообще говоря, актуален вопрос об эффективизации (см., например, [9]) формул, задающих ее решение. 
Таким образом, несмотря на значительный прогресс в интегрировании гамильтоновых систем вполне обоснована необходимость поиска дополнительных средств исследования с более удобным аналитическим формализмом. В связи с этим мы возвращаемся к работе [10], в которой используются весьма простые технические средства, и показываем, что анализ проблемы с точки зрения аналитических свойств решений гамильтоновых систем позволяет ответить на ряд принципиальных вопросов, которые оказываются вне поля зрения в схеме Адлера-Мербеке-Реймана-Семенова-Тян-Шанского (см. [11]).

Для исследования аналитических свойств решений квазиоднородных гамильтоновых систем мы используем идею работы [12] факторизации фазового потока по квазиоднородному растяжению. В результате факторизации фазовое пространство отображается на компактное голоморфное многообразие со структурой голоморфного слоения с особенностями, которые соответствуют особым точкам решений рассматриваемой задачи.

Компактность профакторизованного фазового пространства позволяет получить полную классификацию особых точек, благодаря которой оказывается возможным доказать теорему об однозначности решений многомерного случая Лагранжа и найти полный список целых решений.

Схема доказательства этих результатов точно такая же, как и при получении аналогичных результатов для классической задачи о движении тяжелого твердого тела, полученных в [12] и [13]. Чтобы сделать настоящую статью максимально понятной, мы приводим не только необходимые результаты этих статей, но и идеи их доказательств.

\section{§ 2. Формулировка основных результатов}

Многомерное обобщение случая Лагранжа задачи о движении тяжелого твердого тела рассматриваем в следующем виде:

$$
\left\{\begin{array}{l}
\dot{M}=[M, \Omega]+R \wedge \Gamma, \\
\dot{\Gamma}=-\Omega \Gamma
\end{array}\right.
$$

здесь $M, \Omega \in \operatorname{so}(n), \Gamma, R \in \mathbb{R}^{n}$. Матрица $\Omega$ есть матрица угловой скорости в теле, оператор инерции имеет вид $M=\Omega I+I \Omega, I^{T}=I ; \Gamma-$ вектор силы тяжести в теле и $R$ - вектор центра тяжести.

Теорема 1. Все решения многомерного обобщения случая Лагранжа задачи о движении тяжелого твердого тела однозначны.

ЗАмечАниЕ 1. Однозначность решений многомерного обобщения случая Лагранжа не является тривиальным фактом, поскольку линеаризация потока происходит на якобиане кривой, вообще говоря, более, чем 4-го порядка. Как известно (см. [6]), обращение гиперэллиптического интеграла является неоднозначной функцией. 
Подобный нетривиальный факт имеет место и в классическом случае Ковалевской, решение которого выражается через гиперэллиптические (ультраэллиптические) интегралы, но при этом является однозначным при всех начальных данных.

Как доказано Архангельским (см. [6]) на примере задачи о движении тяжелого твердого тела с ньютоновским потенциалом, из полной интегрируемости по Лиувиллю не обязательно следует однозначность решений. Тем не менее, по-видимому, существуют некоторые естественные причины, в силу которых решения интегрируемых задач, как правило, являются однозначными функциями времени на комплексной плоскости.

ТЕОРема 2. Все целые решения многомерного обобщения случая Лагранжа задачи о движении тяжелого твердого тела выражаются через круговые функции времени.

\section{§ 3. Гамильтонов формализм на алгебрах Ли}

Как уже было отмечено, интегрирование гамильтоновых систем, особенно высокой размерности, требует наличия у исследуемой задачи достаточно богатой группы симметрий. Как следует из схемы интегрирования методом LA-пары с параметром, весьма желательно, чтобы задача была поставлена не просто на симплектическом многообразии (см., например, [14]), а на орбите коприсоединенного представления (см. [15]) подходящей алгебры Ли.

Прежде чем перейти к такому способу задания гамильтоновой системы, поставим задачу на кокасательном расслоении группы движений данной системы, например задачу о движении тяжелого твердого тела.

Как известно (см. [14]), на каждом кокасательном расслоении гладкого многообразия имеется каноническая 1-форма $\alpha=p d q$ ( $p$ - координата ковектора из кокасательного расслоения, $q$ - координата на многообразии), дифференциал которой есть каноническая симплектическая форма $\stackrel{2}{\omega}=d \alpha$. Множество всех состояний системы описывается элементами $g$ группы $\mathfrak{G}$ движений системы. Кинетическая энергия есть квадратическая форма на кокасательном расслоении группы, инвариантная относительно действия группы. Поскольку кокасательное расслоение группы Ли тривиально, каждый элемент этого расслоения можно представить в виде $(p, q)$. Тогда кинетическая энергия будет иметь вид $T=\frac{1}{2}\left(p, I^{-1} p\right)$, где $I$ - оператор инерции. Потенциальная энергия $U(q)$ зависит только от состояния системы. Итак, $H=T(p)+U(q)$.

Если бы гамильтониан был левоинвариантным, можно было бы сразу редуцировать задачу на орбиту коприсоединенного представления (см. [16]) группы $\mathfrak{G}$, однако для задачи о движении тяжелого твердого тела это не так, поэтому мы воспользуемся результатом из [17].

При изложении схемы редукции, как правило, используется инвариантный стиль доказательства, который хотя и является достаточно изысканным, к сожалению, существенно сложнее доказательства "в координатах". Желая сделать изложение максимально ясным, мы приводим соответствующий результат с координатным доказательством. 
Теорема 3. Пусть на кокасателъном расслоении $T^{*} \mathfrak{G}$ группъ Ли $\mathfrak{G}$ задан гамильтониан $H=T(p)+U(q)$, где $T(p)$ - кинетическая энергия, являющаяся левоинвариантной частью гамильтониана, $p \in \mathfrak{g}^{*}, \mathfrak{g}$ - алгебра Ли группъ Ли $\mathfrak{G}$ и $U(q), q \in \mathfrak{G},-$ потенииальная энергия.

Тогда гамильтонова система, заданная с помощью гамильтониана $H$, pедуцируется в гамильтонову систему, заданную на орбите коприсоединенного представления алгебры Ли $\widehat{\mathfrak{g}}=\mathfrak{g} \oplus V$, где $V$ - линейное пространство, порожденное действием группь $\mathfrak{G}$ на функиию $U(q)$. Алгебра $\widehat{\mathfrak{g}}$ является полупрямой суммой алгебры $\mathfrak{g}$ и линейного пространства $V$, а действие $\mathfrak{g}$ на $V$ порождается действием группы $\mathfrak{G}$.

ДокАзАТЕЛьство. Найдем сначала скобки Пуассона функций, образующих алгебру Ли, изоморфную $\widehat{\mathfrak{g}}$.

Пусть $X, Y$ - левоинвариантные функции, заданные на $T^{*} \mathfrak{G}$, ограничение которых на $\mathfrak{g}^{*}$ является линейной функцией. Функции $X, Y$ можно отождествить с левоинвариантными векторными полями, и в координатах они будут иметь вид $X(p, q)=p_{i} X^{i}(q), Y(p, q)=p_{i} Y^{i}(q)$. Градиентами этих функций будут соответственно

$$
\operatorname{grad} X(p, q)=\left(X^{j},\left(p_{i} \frac{\partial X^{i}}{\partial q_{j}}\right)\right), \quad \operatorname{grad} Y(p, q)=\left(Y^{j},\left(p_{i} \frac{\partial Y^{i}}{\partial q_{j}}\right)\right) .
$$

Значение формы $\stackrel{2}{\omega}=d p \wedge d q$ будет равно

$$
\stackrel{2}{\omega}(\operatorname{grad} X(p, q), \operatorname{grad} Y(p, q))=X^{j} p_{i} \frac{\partial Y^{i}}{\partial q_{j}}-Y^{j} p_{i} \frac{\partial X^{i}}{\partial q_{j}}=p_{i}[X, Y]^{i},
$$

а это означает, что скобкой Пуассона функций $X, Y$ будет функция $[X, Y]$, т.е. соответствие между левоинвариантными функциями, линейными на $\mathfrak{g}^{*}$, продолжается до изоморфизма алгебр.

Пусть $V_{1}(q), V_{2}(q)$ - функции из $V$. В этом случае $V_{i}(p, q)=V_{i}(q), i=1,2$, и в координатах $(p, q)$

$$
\operatorname{grad}_{T^{*} \mathfrak{G}} V_{i}=\left(0, \operatorname{grad} V_{i}\right), \quad i=1,2 .
$$

Очевидно, что теперь $\left\{V_{1}(q), V_{2}(q)\right\}=0$.

Наконец, для функций $X(p, q), V(q)$ имеем

$$
\stackrel{2}{\omega}(\operatorname{grad} X(p, q), \operatorname{grad} V(q))=X^{i} \frac{\partial V}{\partial q_{i}}=L_{X} V ;
$$

здесь $L_{X} V$ - производная функции $V$ вдоль векторного поля $X$.

Итак, рассмотренные выше функции образуют алгебру Ли, изоморфную $\widehat{\mathfrak{g}}$. Зная скобки Пуассона базисных функций этой алгебры, мы получаем скобку Пуассона на множестве полиномов от базисных функций. В это множество включается и гамильтониан. Наконец, функции, являющиеся инвариантами коприсоединенного представления, становятся первыми интегралами рассматриваемой задачи. Теорема доказана. 
Для многомерного обобщения кинетическая энергия твердого тела, состоящего из дискретного числа материальных точек, равна

$$
T=\frac{1}{2} \sum_{i} m_{i}\left(\dot{A} r_{i}, \dot{A} r_{i}\right)=\frac{1}{2} \sum_{i} m_{i}\left(\Omega r_{i}, \Omega r_{i}\right)=\frac{1}{2} \sum_{i} m_{i} \omega_{k l} r_{i l} \omega_{k m} r_{i m}
$$

здесь $A \in \mathrm{So}(n), \dot{A}=A \Omega, \Omega \in \mathrm{so}(n)-$ скорость в теле. Для сплошного тела надо взять соответствующий интеграл. Обозначим $I_{l m}=\sum_{i} m_{i} r_{i l} r_{i m}$, тогда $T=-\frac{1}{2} \operatorname{Tr}(\Omega I \Omega) ;$ при этом момент в теле равен $M=\frac{\partial T}{\partial \Omega}=-\frac{1}{2}(\Omega I+I \Omega)$. Для простоты коэффициент перед $M$ внесем в значение $I$ и будем иметь $M=$ $\Omega I+I \Omega$.

Таким образом, уравнения Эйлера $\dot{m}=\operatorname{ad}_{\operatorname{grad} T(m)}^{*} m$ в матричном виде записываются следующим образом (см. [18]):

$$
(\Omega I+I \Omega)^{\cdot}=[\Omega I+I \Omega, \Omega]=\left[I, \Omega^{2}\right],
$$

и именно это представление было проинтегрировано в [1].

С помощью скобки Пуассона эти же уравнения можно получить следующим образом.

Определим базисные векторы в so $(n): \nu_{i j}$ - это кососимметрическая матрица, у которой на $(i, j)$-м, и $(j, i)$-м местах стоят соответственно 1 и -1 . В таких обозначениях имеем $\left\{\nu_{i j}, \nu_{j k}\right\}=\nu_{i k}$.

$\mathrm{K}$ этим обозначениям добавим $I=\operatorname{diag}\left(\alpha_{1}, \ldots, \alpha_{n}\right), \nu_{i j}=\left(\alpha_{i}+\alpha_{j}\right) \omega_{i j}$; тогда

$$
T=\frac{1}{2} \sum_{i<j} \frac{\nu_{i j}^{2}}{\alpha_{i}+\alpha_{j}}
$$

а искомая гамильтонова система $\dot{\nu}_{i j}=\left\{\nu_{i j}, H\right\}$ будет иметь вид в полном соответствии с матричной записью:

$$
\left(\alpha_{i}+\alpha_{j}\right) \dot{\omega}_{i n}=\left(\alpha_{i}-\alpha_{j}\right) \sum_{l=1}^{n} \omega_{i l} \omega_{l j}
$$

Теперь для получения многомерного обобщения задачи о движении тяжелого твердого тела надо выбрать подходящее представление для группы Ли $\operatorname{So}(n)$. Эта задача решается не однозначно.

ЗАмЕчАниЕ 2. Для многомерного обобщения задачи о движении тяжелого твердого тела в настоящей работе рассматривается минимальное представление $\mathrm{So}(n): \mathbb{R}^{n} \rightarrow \mathbb{R}^{n}$ (см. также [11]). В работе [19] выбирается представление $\operatorname{So}(n): \operatorname{So}(n) \rightarrow \operatorname{So}(n)$.

Определим базисные векторы алгебры $\widehat{\mathfrak{g}}=\operatorname{so}(n) \oplus \mathbb{R}^{n}: \nu_{i j}-$ это кососимметрическая матрица, у которой на $(i, j)$-м и $(j, i)$-м местах стоят соответственно 1 и $-1, \gamma_{i}$ - столбец, имеющий на $i$-м месте 1 . В таких обозначениях имеем

$$
\left\{\nu_{i j}, \nu_{j k}\right\}=\nu_{i k}, \quad\left\{\nu_{i j}, \gamma_{j}\right\}=\gamma_{i}, \quad\left\{\gamma_{i}, \gamma_{j}\right\}=0
$$


Выбирая линейный потенциал по $\gamma_{i}$, получаем

$$
H=\frac{1}{2} \sum_{i<j} \frac{\nu_{i j}^{2}}{\alpha_{i}+\alpha_{j}}+\sum_{i} r_{i} \gamma_{i},
$$

а искомая гамильтонова система

$$
\left\{\begin{array}{l}
\dot{\nu}_{i j}=\left\{H, \nu_{i j}\right\} \\
\dot{\gamma}_{i}=\left\{H, \gamma_{i}\right\}
\end{array}\right.
$$

будет иметь вид

$$
\left\{\begin{array}{l}
\left(\alpha_{i}+\alpha_{j}\right) \dot{\omega}_{i j}=\left(\alpha_{i}-\alpha_{j}\right) \sum_{l=1}^{n} \omega_{i l} \omega_{l j}+\gamma_{j} r_{i}-\gamma_{i} r_{j} \\
\dot{\gamma}_{i}=-\sum_{l=1}^{n} \omega_{i l} \gamma_{l} .
\end{array}\right.
$$

В терминах $\mathrm{ad}^{*}$ имеем

$$
\left\{\begin{array}{l}
\dot{m}=\operatorname{ad}_{\operatorname{grad} T(m)}^{*} m+\operatorname{ad}_{\operatorname{grad} U(x)}^{*} x, \\
\dot{x}=\operatorname{ad}_{\operatorname{grad} T(m)}^{*} x ;
\end{array}\right.
$$

здесь $m \in \operatorname{so}(n)^{*}, x \in\left(\mathbb{R}^{n}\right)^{*}$, или, в матричном виде,

$$
\left\{\begin{array}{l}
\dot{M}=[M, \Omega]+R \wedge \Gamma \\
\dot{\Gamma}=-\Omega \Gamma
\end{array}\right.
$$

здесь $M, \Omega \in \operatorname{so}(n), \Gamma, R \in \mathbb{R}^{n}$. Матрица $\Omega$ есть матрица угловой скорости в теле, оператор инерции имеет вид $M=\Omega I+I \Omega, I^{T}=I, \Gamma-$ вектор силы тяжести в теле и $R$ - вектор центра тяжести.

\section{§4. Интегрируемость по Лиувиллю многомерного обобщения случая Лагранжа}

Естественно считать, что случай Лагранжа имеет место, если предположить, что подобно классическому случаю все диагональные элементы оператора инерции, кроме одного, равны между собой и все координаты вектора силы тяжести, кроме одного, равны нулю, т.е. если $I=\operatorname{diag}(A, \ldots, A, B)$, $R=\left(0, \ldots, 0, r_{n}\right)$, то система (1) будет иметь достаточно простой вид

$$
\left\{\begin{array}{l}
\dot{\omega}_{i j}=0, i, j \leqslant n-1, \\
\dot{\omega}_{i n}=\frac{A-B}{A+B} \sum_{l=1}^{n} \omega_{i l} \omega_{l n}+\frac{r_{n}}{(A+B)} \gamma_{i}, i \leqslant n-1, \\
\dot{\gamma}_{i}=-\sum_{l=1}^{n} \omega_{i l} \gamma_{l} .
\end{array}\right.
$$


ЗАмЕчАниЕ 3. Предложенное многомерное обобщение случая Лагранжа является корректным в том смысле, что при $n=3$ система (1) совпадает с классической системой уравнений Эйлера-Пуассона, а система (2) описывает классический случай Лагранжа.

При этом нельзя заранее утверждать, что не существует другого обобщения, для которого уравнения (2) являются частным случаем.

Учитывая, что часть переменных $\omega_{i j}$ являются формальными, запишем уравнения (2) в окончательном виде следующим образом:

$$
\left\{\begin{array}{l}
\dot{p}=\lambda \Omega p+\mu \gamma \\
\dot{\gamma}=-\Omega \gamma-x p \\
\dot{x}=(p, \gamma)
\end{array}\right.
$$

здесь $p=\left(\nu_{1 n}, \ldots, \nu_{n-1, n}\right), \gamma=\left(\gamma_{1}, \ldots, \gamma_{n-1}\right), x=\gamma_{n}, \Omega=\left(\omega_{i j}\right), 1 \leqslant i, j \leqslant n-1$, $\lambda=\frac{A-B}{A+B}, \mu=\frac{r_{n}}{A+B}$.

Далее будем называть многомерной задачей Лагранжа дифференциальные уравнения, задающие обобщение случая Лагранжа для многомерного тела.

ПРЕДЛОЖЕНИЕ 1 (см. [10]). Пусть кососимметрическая матрица U коммутирует с кососимметрической матрищей $\Omega$. Тогда существует первый интеграл $J_{U}$ системы $(2)$,

$$
J_{U}=(\lambda+1)(\Omega U p, p)-2 \mu(U p, \gamma)
$$

Доказательство этого представляет собой простое вычисление производной функции $J_{U}$ вдоль векторного поля (3).

Полученные интегралы определены не на всей алгебре, поэтому необходимо распространить их на все пространство $\left(\operatorname{so}(n) \oplus \mathbb{R}^{n}\right)^{*}$. Естественный способ, с помощью которого можно это сделать, - взять в качестве матрицы $U$ нечетные степени матрицы $\Omega$. Итак, первыми интегралами задачи (2) являются функции

$$
J_{r}=(\lambda+1)\left(\Omega^{2 r} p, p\right)-2 \mu\left(\Omega^{2 r-1} p, \gamma\right), \quad r=1, \ldots,\left[\frac{n-1}{2}\right] .
$$

Теорема 4 (см. [10]). Для почти всех началъных условий в задаче, заданной на орбитах коприсоединенного представления алгебры Ли $\operatorname{so}(n) \oplus \mathbb{R}^{n}$ со структурой, определенной соотношениями

$$
\left\{\nu_{i j}, \nu_{j k}\right\}=\nu_{i k}, \quad\left\{\nu_{i j}, \gamma_{j}\right\}=\gamma_{i}, \quad\left\{\gamma_{i}, \gamma_{j}\right\}=0
$$

и гамильтонианом

$$
H=\frac{1}{4 A} \sum_{i<j<n-1} \nu_{i j}^{2}+\frac{1}{2(A+B)} \sum_{i<n-1} p_{i}^{2}+r_{n} \gamma_{n}, \quad \nu_{i n}=(A+B) p_{i},
$$

гамильтонова система sgrad $H$ является вполне интегрируемой по Лиувиллю (см. [14]) и в смысле некоммутативной интегрируемости (см. [20]). Все 
решения рассматриваемой задачи являются условно-периодическими, а траектории движения лежат на инвариантных торах размерности $n-1$. Эти торы являются поверхностями уровня гамильтониана $H$, первых интегралов

$$
\Omega_{i j}=\frac{\nu_{i j}}{2 A}, \quad i<j<n-1,
$$

образующих алгебру $\mathfrak{h} \approx \mathrm{so}(n-1)$, и первых интегралов

$$
J_{r}=(\lambda+1)\left(\Omega^{2 r} p, p\right)+2 \mu\left(\Omega^{2 r-1} p, \gamma\right), \quad r=1, \ldots,\left[\frac{n-1}{2}\right] .
$$

В схеме интегрируемости по Лиувиллю инвариантные торы половинной размерности орбиты коприсоединенного представления задаются гамильтонианом $H$, первыми интегралами

$$
J_{r}, \quad r=1, \ldots,\left[\frac{n-1}{2}\right],
$$

и сдвигами инвариантов алгебры линейных интегралов

$$
\operatorname{Tr}(\Omega+\lambda a)^{2 r}, \quad r=1, \ldots,\left[\frac{n-1}{2}\right], \quad \lambda \in \mathbb{R}, \quad a \in \mathfrak{h} .
$$

ДокАЗАТЕЛЬСтво. Инволютивность первых интегралов $J_{r}, r=1, \ldots,\left[\frac{n-1}{2}\right]$, доказывается непосредственным вычислением. В качестве промежуточных результатов вычисления укажем следующие.

Пусть $h \in \mathfrak{h}$; обозначим через $\Omega_{h}$ матрицу, равную $(\Omega, h)$. Тогда

$$
\{h, \Omega\}=\left[\Omega, \Omega_{h}\right], \quad\{h, p\}=-\Omega_{h} p, \quad\{h, \gamma\}=-\Omega_{h} \gamma
$$

и, как следствие,

$$
\left\{h, \Omega^{r} p\right\}=-\Omega_{h} \Omega^{r} p, \quad\left\{h, \Omega^{r} \gamma\right\}=-\Omega_{h} \Omega^{r} \gamma .
$$

Кроме того, обозначим $J_{r}^{\prime}=\left(\Omega^{2 r} p, p\right), J_{r}^{\prime \prime}=\left(\Omega^{2 r-1} p, \gamma\right)$; тогда

$$
\left\{h, J_{r}^{\prime}\right\}=\left\{h, J_{r}^{\prime \prime}\right\}=0 .
$$

Используя приведенные соотношения, легко получить

$$
\left\{J_{r}^{\prime}, J_{s}^{\prime}\right\}=\left\{J_{r}^{\prime \prime}, J_{s}^{\prime \prime}\right\}=0, \quad\left\{J_{r}^{\prime}, J_{s}^{\prime \prime}\right\}+\left\{J_{r}^{\prime \prime}, J_{s}^{\prime}\right\}=0,
$$

что доказывает инволютивность интегралов $J_{r}$.

Чтобы доказать функциональную независимость первых интегралов

$$
\Omega_{i j}, \quad i, j \leqslant n=1, \quad J_{r}, \quad r=1, \ldots,\left[\frac{n-1}{2}\right],
$$

на орбите коприсоединенного представления, нужно доказать линейную независимость косых градиентов этих функций в некоторой точке. 
Предположим, что существуют $\lambda_{0}, \lambda_{i j}, \lambda_{m}$ такие, что

$$
\left.\operatorname{sgrad}\left(\sum \lambda_{0} H+\lambda_{i j} \Omega_{i j}+\lambda_{r} I_{r}\right)\right|_{y}=0
$$

Тогда как следствие имеем в точке $y$

$$
\left\{\sum \lambda_{0} H+\lambda_{i j} \Omega_{i j}+\lambda_{r} J_{r}, h\right\}=\left\{\sum \lambda_{i j} \Omega_{i j}, h\right\}=0 .
$$

Кососимметрическую матрицу с элементами $\lambda_{i j}$ обозначим $\Lambda$. Имеем

$$
\operatorname{Tr}(\Lambda\{\Omega, h\})=\operatorname{Tr}\left(\Lambda\left[\Omega_{h}, \Omega\right]\right)=\operatorname{Tr}\left(\Omega_{h}[\Omega, \Lambda]\right)=0 \quad \Longrightarrow \quad[\Omega, \Lambda]=0,
$$

т.е. $\Lambda$ есть линейная комбинация степеней $\Omega$ почти всюду.

Предположим, что в точке $y$ матрица $\Omega$ не имеет кратных корней, $\Omega p \neq 0$, $\gamma=0$ и $x \neq 0$. Тогда в точке $y$ имеем

$$
\left\{J_{r}, \gamma\right\}=-\frac{2 x}{k+1} \Omega^{2 r} p, \quad\{H, \gamma\}=-x p, \quad\{\Omega, \gamma\}=0 .
$$

Нетрудно видеть, что когда $n$ нечетно, $\operatorname{sgrad} J_{r}, r=1, \ldots,\left[\frac{n-1}{2}\right]$, независимы, а когда $n$ четно, независимы $\operatorname{sgrad} J_{r}, r=1, \ldots,\left[\frac{n-1}{2}\right], \operatorname{sgrad} H$. Следовательно,

$$
\left.\operatorname{sgrad}\left(\sum \lambda_{0} H+\lambda_{i j} \Omega_{i j}+\lambda_{r} J_{r}\right)\right|_{y}=0 \quad \Longrightarrow \quad \lambda_{r}=0, \quad \lambda_{0}=0
$$

(в случае нечетного $n$ гамильтониан не рассматриваем). Далее,

$$
\left\{\lambda_{i j} \Omega_{i j}, p\right\}=\operatorname{Tr}(\Lambda\{\Omega, p\})=-2 \Lambda p \neq 0,
$$

т.е. найдена точка, где функции $\Omega_{i j}, i, j \leqslant n-1, J_{r}, r=1, \ldots,\left[\frac{n-1}{2}\right]$, функционально независимы на орбите, а если $n$ четно, то в набор этих независимых функций можно добавить и гамильтониан.

Размерность орбиты общего положения коприсоединенного представления алгебры Ли so $(n) \oplus \mathbb{R}^{n}$ равна $\frac{n(n+1)}{2}-\left[\frac{n-1}{2}\right]-1$ (см. [21]), следовательно, размерность поверхности уровня первых интегралов равна $n-1$.

Теперь необходимо указать $n-1$ коммутирующих векторных полей на поверхности уровня первых интегралов. Это значит, что нужно найти функции, находящиеся в инволюции со всеми первыми интегралами. Очевидно, что этими функциями являются интегралы $J_{r}$, инварианты $\operatorname{Tr}\left(\Omega^{2 r}\right), r=1, \ldots,\left[\frac{n-1}{2}\right]$, коприсоединенного представления алгебры линейных интегралов $\mathfrak{h}$, а если $n$ четно, то и $H$. Как видим, этих функций достаточное количество, равное $n-1$, следовательно, инвариантные многообразия - торы, поток на которых условно-периодичен.

Инвариантные торы размерности $n-1$ можно различными способами сгруппировать в инвариантные торы половинной размерности задачи. Для этого некоммутативную алгебру первых интегралов надо заменить инволютивным набором функционально независимых интегралов в количестве половинной размерности орбиты коприсоединенного представления. Для алгебры $\mathrm{so}(n)$, в частности, эта задача решена в [2]. Как следует из этой работы, в качестве 
инволютивного набора можно взять, например, сдвиги инвариантов коприсоединенного представления; при этом количество функционально независимых функций, находящихся в инволюции, будет достаточным. Тем самым, теорема полностью доказана ${ }^{1}$.

\section{§5. Классификация особых точек решений и целые решения классической задачи о движении тяжелого твердого тела}

Содержание этого параграфа есть краткое изложение необходимых нам фактов из [12].

Уравнения Эйлера-Пуассона, задающие решение классической задачи о движении тяжелого твердого тела, запишем в следующем виде:

$$
\left\{\begin{array}{l}
A \dot{p}=A p \times p+\gamma \times r, \\
\dot{\gamma}=\gamma \times p
\end{array}\right.
$$

здесь $p=\left(p_{1}, p_{2}, p_{3}\right) \in \mathbb{C}^{3}, \gamma=\left(\gamma_{1}, \gamma_{2}, \gamma_{3}\right) \in \mathbb{C}^{3}, A p=\left(A_{1} p_{1}, A_{2} p_{2}, A_{3} p_{3}\right), A_{i}>0$, $r=\left(r_{1}, r_{2}, r_{3}\right) \in \mathbb{R}^{3}$.

Сделаем замену переменных следующего вида:

$$
p(t)=e^{-\tau} \widetilde{p}(\tau), \quad \gamma(t)=e^{-2 \tau} \widetilde{\gamma}(\tau) ;
$$

получим

$$
\left\{\begin{array}{l}
A \dot{\tilde{p}}=A \widetilde{p} \times \widetilde{p}+\widetilde{\gamma} \times r+A \widetilde{p}, \\
\dot{\widetilde{\gamma}}=\widetilde{\gamma} \times \widetilde{p}+2 \widetilde{\gamma} .
\end{array}\right.
$$

Связь между решениями (4) и (5) выражается соотношениями

$$
p(t)=\frac{1}{t-t_{*}} \widetilde{p}\left(\ln \left(t-t_{*}\right)\right), \quad \gamma(t)=\frac{1}{\left(t-t_{*}\right)^{2}} \widetilde{\gamma}\left(\ln \left(t-t_{*}\right)\right) .
$$

Пусть теперь $\left(\widetilde{p}^{0}, \widetilde{\gamma}^{0}\right)$ - особая точка системы дифференциальных уравнений $(5)$; тогда траектория $\widetilde{p}(\tau), \widetilde{\gamma}(\tau)$, входящая в $\left(\widetilde{p}^{0}, \widetilde{\gamma}^{0}\right)$, при обратной замене задается решением с особой точкой $t_{*}\left(t-t_{*}=e^{\tau}\right)$

$$
p(t)=\widetilde{p}^{0}\left(t-t_{*}\right)^{-1}+\cdots, \quad \gamma(t)=\widetilde{\gamma}^{0}\left(t-t_{*}\right)^{-2}+\cdots .
$$

Теперь чтобы получить асимптотику особых точек решений исходной системы (4), достаточно получить асимптотику решений системы (5) в окрестности особых точек, что является стандартной задачей. Для этого нужно сначала решить линеаризованную систему в окрестности особой точки, а затем применить оператор Пикара для полученного первого приближения.

ОПРедЕЛЕНиЕ 1. Алгебраическую систему, задающую особые точки системы (5), мы называем характеристической:

$$
\left\{\begin{array}{l}
A \widetilde{p}^{0} \times \widetilde{p}^{0}+\widetilde{\gamma}^{0} \times r+A \widetilde{p}^{0}=0, \\
\widetilde{\gamma}^{0} \times \widetilde{p}^{0}+2 \widetilde{\gamma}^{0}=0 .
\end{array}\right.
$$
[23].

${ }^{1}$ Результаты, обобщающие теорему 4, см., например, в работе [11], а также в работах [22], 
ЗАмечАниЕ 4. Характеристическая система для уравнений Эйлера-Пуассона полностью решается, если выполнено условие вырожденности: хотя бы одна координата $r_{i}$ равна нулю или среди собственных значений оператора инерции $A_{i}$ есть равные.

В невырожденном случае система решается, если известны корни некоторого полинома восьмой степени с коэффициентами, зависящими от $A_{i}, r_{i}$.

При решении характеристической системы обнаруживаются случаи ее вырождения, соответствующие известным случаям интегрируемости Эйлера, Лагранжа, Ковалевской и Гриоли.

ОПРЕДЕЛЕНиЕ 2. Характеристическая система для уравнений ЭйлераПуассона имеет два типа корней: $\left(\widetilde{p}^{0}, 0\right)$ и $\left(\widetilde{p}^{0}, \widetilde{\gamma}^{0}\right), \widetilde{\gamma}^{0} \neq 0$. Эти корни являются коэффициентами главных частей асимптотик двух типов особых точек решений уравнений Эйлура-Пуассона, которые мы будем называть $\alpha$-особыми и $\beta$-особъми точками.

Нахождение асимптотик особых точек решений уравнений Эйлера-Пуассона вышеописанным способом не гарантирует получения полного списка асимптотик всех особых точек. Для того чтобы найти все особые точки, мы применяем факторизацию потока в фазовом пространстве, которая превращает фазовое пространство в компактное голоморфное многообразие со структурой голоморфного слоения с особыми точками. Исследование всех особых точек полученного слоения позволяет найти все особые точки решений уравнений Эйлера-Пуассона.

Пусть $p(t), \gamma(t)$ - решение уравнений Эйлера-Пуассона; тогда решением будет и $\alpha p(\alpha t), \alpha^{2} \gamma(\alpha t)$. Это обстоятельство позволяет произвести факторизацию на множестве траекторий решений системы (4).

Теорема 5. Пусть $\mathbb{C}$ действует в $\mathbb{C}^{n}$ следующим образом:

$$
\alpha:\left(z_{1}, \ldots, z_{n}\right) \rightarrow\left(\alpha^{k_{1}} z_{1}, \ldots, \alpha^{k_{n}} z_{n}\right), \quad k=\left(k_{1}, \ldots, k_{n}\right) \in \mathbb{N}^{n} .
$$

Тогда факторпространство $P_{k}^{n-1}=\left\{\left(z_{1}^{\left(k_{1}\right)}: \ldots: z_{n}^{\left(k_{n}\right)}\right)\right\}$ по этому действию является компактным голоморфным многообразием.

Теорема 6. Каноническая проекиия $\pi: \mathbb{C}^{6} \rightarrow P_{*}^{5}, *=(1,1,1,2,2,2), \mathbb{C}^{6}=$ $\{(p, \gamma)\}$, проектирует слоение, задаваемое потоком (4) в голоморбное слоение $\mathscr{F}$ компактного голоморфного многообразия $P_{*}^{5}$.

Все особие точки слоения Я્F компактного голоморфного многообразия $P_{*}^{5}$ есть $\pi$-проекиии особъх точек $z_{0}$ системы уравнений (4) и т-проекиии решений $\tilde{z}^{0}$ характеристической системы.

ЗАмЕчАниЕ 5. Слоение $\mathscr{F}$ является интегрируемым, поскольку существует послойно-инвариантное отображение

$$
\begin{gathered}
J: P_{*}^{5} \backslash X^{2} \rightarrow P^{2}, \\
J:\left(p_{1}^{(1)}: p_{2}^{(1)}: p_{3}^{(1)}: \gamma_{1}^{(2)}: \gamma_{2}^{(2)}: \gamma_{3}^{(2)}\right) \rightarrow\left(\mathscr{H}^{6}(p, \gamma): \mathscr{M}^{4}(p, \gamma): \mathscr{T}^{3}(\gamma)\right),
\end{gathered}
$$

где $X^{2}=\{\pi(z): \mathscr{H}(z)=\mathscr{M}(z)=\mathscr{T}(z)=0\}$.

Кроме того, поверхность $X^{2}$ является инвариантной для слоения $\mathscr{F}$. 
ТЕОРема 7. Все особые точки решений уравнений Эйлера-Пуассона (4) являттся $\alpha$ - или $\beta$-особыми точками либо являются предельными особыми точками $\alpha$ - или $\beta$-особых точек, либо аппроксимируются асимптотикой $\beta$-особых точек (последнее, если одно из собственных значений линейной аппроксимачии в окрестности особой $\beta$-точки системы (5) равно нулю).

В формулировке теоремы 7 отсутствует упоминание об особых точках, являющихся проекциями особых точек уравнений Эйлера-Пуассона (4). Причина заключается в том, что эти особые точки дают асимптотику при $t \rightarrow t_{*}=\infty$, в отличие от $\alpha$ - или $\beta$-особых точек, для которых $t_{*} \in \mathbb{C}$. Для доказательства теоремы существенна только компактность многообразия $P_{*}^{5}$.

ТеОрема 8. Все непостоянные решения z $(t)$ уравнений Эйлера-Пуассона имеют особъе точки $t_{*} \in \mathbb{C}$, если

$$
\prod_{\sigma} B_{12} \sum_{\sigma} r_{1} \sqrt{B_{23}} \neq 0
$$

Условие теоремы 8 находится как условие отсутствия особых точек, являющихся $\pi$-проекциями особых точек уравнений (4). Идея доказательства заключается в следующем.

Пусть $\Gamma(t)$ - траектория, по которой $z(t) \rightarrow \infty$. Благодаря компактности $P_{*}^{5}$ на этой траектории найдутся точки $t_{0}, t_{1}$ такие, что $\pi\left(z\left(t_{0}\right)\right) \approx \pi\left(z\left(t_{1}\right)\right)$. Пусть норма вектора $z\left(t_{1}\right)$ в $k>0$ раз больше нормы $z\left(t_{0}\right)$. Тогда в силу квазиоднородности уравнений Эйлера-Пуассона и отсутствия особых точек, являющихся $\pi$-проекциями особых точек уравнений (4), от точки $t_{1}$ можно дойти до точки $t_{2}$ так, что норма вектора $z\left(t_{2}\right)$ будет в $k$ раз больше нормы $z\left(t_{1}\right)$, причем за время, в $k$ раз меньшее, чем от $t_{0}$ до $t_{1}$. Повторяя это действие бесконечное число раз, за конечное время можно достичь бесконечного значения нормы $z(t)$.

Теорема 9. Все целье решения уравнений Эйлера-Пуассона (4) исчерпьваются частными решениями случаев Эйлера, Лагранжса и Гриоли.

Теперь (см. теорему 8) мы можем, используя асимптотику особых точек $\pi\left(z_{0}\right)$, найти все целые решения.

\section{§6. Асимптотика особых точек решений многомерного обобщения случая Лагранжа}

Для нахождения асимптотики, следуя методу из [12], сделаем замену переменных в системе (3) следующего вида:

$$
\widetilde{\omega}_{i j}(\tau)=e^{\tau} \omega_{i j}\left(e^{\tau}\right), \quad \widetilde{\gamma}_{i}(\tau)=e^{2 \tau} \gamma_{i}\left(e^{\tau}\right) .
$$

Мы получим систему

$$
\left\{\begin{array}{l}
\dot{\tilde{\omega}}_{i j}=\widetilde{\omega}_{i j}, i, j \leqslant n-1, \\
\dot{\tilde{\omega}}_{i n}=\frac{A-B}{A+B} \sum_{l=1}^{n} \widetilde{\omega}_{i l} \widetilde{\omega}_{l n}-\frac{r_{n}}{A+B} \widetilde{\gamma}_{i}+\widetilde{\omega}_{i n}, i \leqslant n-1, \\
\dot{\widetilde{\gamma}}_{i}=-\sum_{l=1}^{n} \widetilde{\omega}_{i l} \widetilde{\gamma}_{l}+2 \widetilde{\gamma}_{i},
\end{array}\right.
$$


для которой исходные переменные выражаются через новые следующим обра3ом:

$$
\omega_{i j}(\tau)=t^{-1} \widetilde{\omega}_{i j}(\ln (t)), \quad \gamma_{i}(\tau)=t^{-2} \widetilde{\gamma}_{i}(\ln (t))
$$

Из этого соотношения следует, что если получить асимптотику траектории, входящей в особую точку системы (6), то в исходных координатах мы получим асимптотику особой точки решения многомерной задачи Лагранжа.

Особые точки системы (6) находим из следующей системы, которую далее называем характеристической:

$$
\left\{\begin{array}{l}
\widetilde{\omega}_{i j}=0, i, j \leqslant n-1, \\
\frac{A-B}{A+B} \sum_{l=1}^{n} \widetilde{\omega}_{i l} \widetilde{\omega}_{l n}-\frac{r_{n}}{A+B} \widetilde{\gamma}_{i}+\widetilde{\omega}_{i n}=0, i \leqslant n-1, \\
-\sum_{l=1}^{n} \widetilde{\omega}_{i l} \widetilde{\gamma}_{l}+2 \widetilde{\gamma}_{i}=0 .
\end{array}\right.
$$

Система (7), записанная в обозначениях, аналогичных (5), имеет вид

$$
\left\{\begin{array}{l}
\mu \widetilde{\gamma}+\widetilde{p}=0 \\
-\widetilde{x} \tilde{p}+2 \widetilde{\gamma}=0 \\
(\widetilde{p}, \widetilde{\gamma})+2 \widetilde{x}=0,
\end{array}\right.
$$

легко решается, и мы получаем $(n-2)$-параметрическую систему решений

$$
\widetilde{x}^{0}=-\frac{2}{\mu}, \quad \widetilde{\gamma}^{0}=-\frac{\widetilde{p}^{0}}{\mu}, \quad\left(\widetilde{p}^{0}, \widetilde{p}^{0}\right)=-4 .
$$

Теперь в найденных особых точках линеаризуем систему дифференциальных уравнений (6):

$$
\left\{\begin{array}{l}
\dot{\tilde{\omega}}_{i j}=\widetilde{\omega}_{i j}, i, j \leqslant n-1, \\
\dot{\widetilde{\omega}}_{i n}=\frac{A-B}{A+B} \sum_{l=1}^{n} \widetilde{\omega}_{i l} \widetilde{\omega}_{l n}^{0}-\frac{r_{n}}{A+B} \widetilde{\gamma}_{i}+\widetilde{\omega}_{i n}, i \leqslant n-1, \\
\dot{\widetilde{\gamma}}_{i}=-\sum_{l=1}^{n}\left(\widetilde{\omega}_{i l} \widetilde{\gamma}_{l}^{0}+\widetilde{\omega}_{i l}^{0} \widetilde{\gamma}_{l}\right)+2 \widetilde{\gamma}_{i},
\end{array}\right.
$$

и запишем ее в обозначениях, аналогичных (5):

$$
\left\{\begin{array}{l}
\dot{\widetilde{\Omega}}=\widetilde{\Omega} \\
\dot{\tilde{p}}=\lambda \widetilde{\Omega} \widetilde{p}^{0}+\mu \widetilde{\gamma}+\widetilde{p} \\
\dot{\widetilde{\gamma}}=-\widetilde{\Omega} \widetilde{\gamma}^{0}-\widetilde{x}^{0} \widetilde{p}-\widetilde{x} \widetilde{p}^{0}+2 \widetilde{\gamma} \\
\dot{\widetilde{x}}=\left(\widetilde{p}^{0}, \widetilde{\gamma}\right)+\left(\widetilde{p}, \widetilde{\gamma}^{0}\right)+2 \widetilde{x}
\end{array}\right.
$$


Система для нахождения собственных значений линейного оператора в правой части (9) имеет вид

$$
\left\{\begin{array}{l}
\widetilde{\Omega}=\nu \widetilde{\Omega} \\
\lambda \widetilde{\Omega} \widetilde{p}^{0}+\mu \widetilde{\gamma}+\widetilde{p}=\nu \widetilde{p} \\
-\widetilde{\Omega} \widetilde{\gamma}^{0}-\widetilde{x} 0 \widetilde{p}-\widetilde{x} \widetilde{p}^{0}+2 \widetilde{\gamma}=\nu \widetilde{\gamma} \\
\left(\widetilde{p}^{0}, \widetilde{\gamma}\right)+\left(\widetilde{p}, \widetilde{\gamma}^{0}\right)+2 \widetilde{x}=\nu \widetilde{x}
\end{array}\right.
$$

Далее возможны два случая: $\widetilde{\Omega}=0$ или $\nu=1$. Рассмотрим их.

1) Для $\nu=1$ имеем

$$
\left\{\begin{array}{l}
\lambda \widetilde{\Omega} \widetilde{p}^{0}+\mu \widetilde{\gamma}=0 \\
-\widetilde{\Omega} \widetilde{\gamma}^{0}-\widetilde{x}^{0} \widetilde{p}-\widetilde{x} \widetilde{p}^{0}+\widetilde{\gamma}=0 \\
\left(\widetilde{p}^{0}, \widetilde{\gamma}\right)+\left(\widetilde{p}, \widetilde{\gamma}^{0}\right)+\widetilde{x}=0 .
\end{array}\right.
$$

Из кососимметричности $\widetilde{\Omega}$, первого уравнения (10) и соотношений (7) следует, что $\left(\widetilde{\gamma}, \widetilde{\gamma}^{0}\right)=0$. Подставим представление $\widetilde{\gamma}$ из первого уравнения (10) и представление $\widetilde{p}$ из второго уравнения (10) в третье уравнение. Мы получаем, что $\widetilde{x}=0$, и, следовательно, решение (10) имеет вид

$$
\widetilde{p}=\frac{\lambda-1}{2} \widetilde{\Omega} \widetilde{p}^{0}, \quad \widetilde{\gamma}=-\frac{\lambda}{\mu} \widetilde{\Omega} \widetilde{p}^{0}, \quad \widetilde{x}=0,
$$

из которого следует, что размерность собственного пространства с собственным значением 1 равна $\operatorname{dim} \widetilde{\Omega}$.

2) Для $\widetilde{\Omega}=0$ имеем

$$
\left\{\begin{array}{l}
\mu \widetilde{\gamma}+\widetilde{p}=\nu \widetilde{p}, \\
-\widetilde{x}^{0} \widetilde{p}-\widetilde{x} \widetilde{p}^{0}+2 \widetilde{\gamma}=\nu \widetilde{\gamma}, \\
\left(\widetilde{p}^{0}, \widetilde{\gamma}\right)+\left(\widetilde{p}, \widetilde{\gamma}^{0}\right)+2 \widetilde{x}=\nu \widetilde{x}
\end{array}\right.
$$

Подставив представление $\widetilde{\gamma}$ из первого уравнение в два других, получим

$$
\left\{\begin{array}{l}
\mu \widetilde{\gamma}=(\nu-1) \widetilde{p} \\
-\mu \widetilde{x} \widetilde{p}^{0}=\nu(\nu-3) \widetilde{p} \\
(\nu-2)\left(\widetilde{p}^{0}, \widetilde{p}\right)=\mu(\nu-2) \widetilde{x}
\end{array}\right.
$$

2а) $\nu(\nu-3) \neq 0$. Выражая $\widetilde{p}$ из второго уравнения и подставляя в третье, с учетом $(8)$ получаем $(\nu-4)(\nu-2)(\nu+1) \mu \widetilde{x}=0$. Итак,

$$
(\nu-4)(\nu-2)(\nu+1)=0, \quad \widetilde{p}=-\frac{\mu \widetilde{x}}{\nu(\nu-3)} \widetilde{p}^{0}, \quad \mu \widetilde{\gamma}=(\nu-1) \widetilde{p} .
$$

Размерность собственного пространства с собственным значением $-1,2$ или 4 равна 1. 
2b) $\nu(\nu-3)=0$. В этом случае мы, очевидным образом, получаем

$$
\widetilde{x}=0, \quad\left(\widetilde{p}^{0}, \widetilde{p}\right)=0, \quad \widetilde{\gamma}=\frac{(\nu-1) \widetilde{p}}{\mu} .
$$

Размерность собственного пространства с собственным значением 0 или 3 равна $n-2$.

Общая размерность собственных пространств равна $2 n-1+\operatorname{dim} \widetilde{\Omega}$, т.е. для линейного оператора системы (9) найден собственный базис.

Теорема 10. Все решения многомерной задачи Лагранжа имеют в особых точках асимптотику следующего вида:

$$
\left\{\begin{aligned}
p(t) & =\widetilde{p}^{0} t^{-1}+\frac{\lambda-1}{2} \Omega \widetilde{p}^{0}+\frac{1}{2} \mu x \widetilde{p}^{0} t-\frac{\lambda}{2} \Omega^{2} \widetilde{p}^{0} t+\frac{1}{4} p^{\perp} t^{2} \\
+ & \frac{1}{8}\left((\lambda-1) \Omega p^{\perp}-2 \mu x_{1} \widetilde{p}^{0}-x^{2} \mu^{2} \widetilde{p}^{0}+2 \mu x \lambda \Omega^{2} \widetilde{p}^{0}-2 \lambda^{2} \Omega^{4} \widetilde{p}^{0}\right) t^{3}+\cdots \\
\gamma(t) & =\widetilde{\gamma}^{0} t^{-2}-\lambda \mu^{-1} \Omega \widetilde{p}^{0} t^{-1}+\frac{1}{2} x \widetilde{p}^{0}-\frac{1}{2} \lambda^{2} \mu^{-1} \Omega^{2} \widetilde{p}^{0} \\
+ & \frac{1}{2} \mu^{-1}\left(p^{\perp}-\lambda \mu x \Omega \widetilde{p}^{0}+\lambda^{2} \Omega^{3} \widetilde{p}^{0}\right) t \\
+ & \frac{1}{8} \mu^{-1}\left((\lambda-3) \Omega p^{\perp}-6 \mu x_{1} \widetilde{p}^{0}-3 \mu^{2} x^{2} \widetilde{p}^{0}+6 x \mu \lambda \Omega^{2} \widetilde{p}^{0}-3 \lambda^{2} \Omega^{4} \widetilde{p}^{0}\right) t^{2}+\cdots \\
x(t) & =\widetilde{x}^{0} t^{-2}+x+x_{1} t^{2}+x_{2} t^{4}+\cdots .
\end{aligned}\right.
$$

В представлении (11)

$$
\widetilde{x}^{0}=-\frac{2}{\mu}, \quad \widetilde{\gamma}^{0}=-\frac{\widetilde{p}^{0}}{\mu}, \quad\left(\widetilde{p}^{0}, \widetilde{p}^{0}\right)=-4, \quad p^{\perp} \perp \widetilde{p}^{0},
$$

$x, x_{1}$ - свободные параметры. Размерность множества свободных параметров равна $2 n-2+\operatorname{dim} \Omega, \Omega \in \mathfrak{h}$. Асимптотика (11) является асимптотикой общего положения.

ДокАЗАТЕЛЬСтво. Используя полученное разложение линейного оператора системы дифференциальных уравнений (9), запишем ее решение для траекторий, входящих в особую точку:

$$
\left\{\begin{array}{l}
\widetilde{p}(t)=\widetilde{p}^{0}+\frac{\lambda-1}{2} \Omega \widetilde{p}^{0} e^{t}+\frac{1}{2} \mu x \widetilde{p}^{0} e^{2 t}+p^{\perp} e^{3 t}+\frac{1}{2} \mu x_{1} \widetilde{p}^{0} e^{4 t} \\
\widetilde{\gamma}(t)=\widetilde{\gamma}^{0}-\lambda \mu^{-1} \Omega \widetilde{p}^{0} e^{t}+\frac{1}{2} x \widetilde{p}^{0} e^{2 t}+2 \mu^{-1} p^{\perp} e^{3 t}+\frac{1}{2} x_{1} \widetilde{p}^{0} e^{4 t} \\
\widetilde{x}(t)=\widetilde{x}^{0}+x e^{2 t}+x_{1} e^{4 t}
\end{array}\right.
$$

Добавляя необходимые слагаемые и возвращаясь к переменным исходной задачи, получаем искомую асимптотику особой точки. Размерность фазового пространства равна $2 n-1+\operatorname{dim} \Omega, \Omega \in \mathfrak{h}$. Следовательно, учитывая число свободных параметров, получаем, что (11) - асимптотика общего положения.

Из доказанной теоремы вытекает следующий результат.

Теорема 11. Все решения многомерного обобщения случая Лагранжа задачи о движении тяжелого твердого тела однозначны. 


\section{§ 7. Целые решения многомерного обобщения случая Лагранжа}

Найдем целые решения для многомерной задачи Лагранжа. Для этого мы используем схему исследования (см. [13]), примененную в классической задаче. Вообще говоря, нет принципиальных препятствий для нахождения всех целых решений не только для многомерного случая Лагранжа, но и для многомерного тела общего вида. Однако соответствующее исследование оказывается весьма громоздким, поскольку требует использования явного вида инвариантов коприсоединенного представления алгебры $\operatorname{so}(n) \oplus \mathbb{R}^{n}$, а затем и решения или хотя бы эффективного исследования нелинейных алгебраических систем уравнений, аналогичных тем, которые рассматривались в классическом случае.

Поскольку задача о движении многомерного тела является не актуальной по существу, a, скорее, модельной, то такие исследования мы не осуществляем, а приводим тот результат, который можно получить без серьезных вычислений, используя лишь идею компактификации фазового пространства.

Целые решения для классической задачи твердого тела имеются, если на слоении, полученном в результате факторизации фазового пространства, есть особые точки, лежащие на нулевой поверхности уровня всех имеющихся первых интегралов. Соответствующие алгебраические соотношения дают переопределенную систему, включающую в себя параметры многомерного тела. Очевидно, что среди решений этой системы должно быть и решение, соответствующее случаю Лагранжа, так как это верно в классическом случае.

Система, задающая подходящие особые точки, имеет вид

$$
\left\{\begin{array}{l}
\lambda \Omega p+\mu \gamma=0 \\
-\Omega \gamma-x p=0 \\
(p, \gamma)=0
\end{array}\right.
$$

Эта система легко решается, и мы имеем

$$
\left\{\begin{array}{l}
\gamma=-\frac{\lambda}{\mu} \Omega p \\
\lambda \Omega^{2} p-\mu x p=0 \\
(p, \Omega p)=0 .
\end{array}\right.
$$

Последнее равенство системы выполняется тождественно, и окончательно получаем

$$
\left\{\begin{array}{l}
\gamma=-\frac{\lambda}{\mu} \Omega p, \\
\left(\lambda \Omega^{2}-\mu x\right) p=0 .
\end{array}\right.
$$

Итак, если мы рассматриваем целое решение, то при $t \rightarrow \infty$, а также при условии $(p, \gamma) \rightarrow \infty$ должны выполняться соотношения

$$
\left\{\begin{array}{l}
\gamma \sim-\frac{\lambda}{\mu} \Omega p, \\
x \sim \frac{\lambda}{\mu},
\end{array}\right.
$$

где $x$ - собственное значение оператора $\left(\Omega^{2}\right)$. 
Последнее означает, что функция $x(t)$ является ограниченной на всей комплексной плоскости времени, т.е. является константой.

Дальнейшие рассуждения являются уже очевидными, поскольку тогда система (3), задающая движение многомерного тела в случае Лагранжа, является просто линейной системой дифференциальных уравнений.

Остается только найти такое решение $(3)$, при котором $(p, \gamma) \equiv 0$. В принципе эта задача алгоритмически разрешима, и можно сначала найти ее общее решение, а потом на полученное решение наложить соответствующее условие. Заранее ясно, что соответствующие вычисления окажутся достаточно громоздкими и, как это обычно бывает, плохо иллюстрируют геометрию решений. Мы поступим иначе.

Поскольку $(p, \gamma) \equiv 0$, то в силу $(3)(p, \gamma)^{\cdot} \equiv 0$, а значит,

$$
(\lambda+1)(\Omega p, \gamma)-x(p, p)+\mu(\gamma, \gamma) \equiv 0
$$

Поскольку функции $(p, p)-2 \mu x,(\gamma, \gamma)+x^{2}$ являются первыми интегралами, то функции $(p, p),(\gamma, \gamma),(\Omega p, \gamma)$ являются константами вдоль искомого решения.

Аналогичным образом получим

$$
(\Omega p, \gamma)^{\cdot}=(\lambda+1)\left(\Omega^{2} p, \gamma\right) \equiv 0, \quad\left(\Omega^{2} \gamma, \gamma\right)^{\cdot}=-x\left(\Omega^{2} p, \gamma\right) \equiv 0
$$

Благодаря наличию интеграла $J_{2}$ получаем также, что $\left(\Omega^{2} p, p\right) \equiv$ const,

$$
\left(\Omega^{2} p, \gamma\right)^{\cdot}=(\lambda+1)\left(\Omega^{3} p, \gamma\right)-x\left(\Omega^{2} p, p\right)+\mu\left(\Omega^{2} \gamma, \gamma\right) \equiv 0
$$

Очевидно, что, действуя таким образом, мы можем доказать, что вдоль искомого целого решения функции $\left(\Omega^{2 r} p, p\right),\left(\Omega^{2 r} p, \gamma\right),\left(\Omega^{2 r+1} p, \gamma\right),\left(\Omega^{2 r} \gamma, \gamma\right)$ являются постоянными. Эти частные интегралы задают поверхность размерности $[(n-1) / 2]$.

В самом деле, рассмотрим градиенты этих функций в $2(n-1)$-мерном пространстве. Очевидно, что градиенты функций

$$
\begin{gathered}
\operatorname{grad}\left(\left(\Omega^{2 r} p, p\right)\right)=\left(2 \Omega^{2 r} p, 0\right), \quad \operatorname{grad}\left(\left(\Omega^{2 r+1} p, \gamma\right)\right)=\left(\Omega^{2 r+1} \gamma, \Omega^{2 r+1} p\right), \\
\operatorname{grad}\left(\left(\Omega^{2 r} p, \gamma\right)\right)=\left(\Omega^{2 r} \gamma, \Omega^{2 r} p\right)
\end{gathered}
$$

линейно независимы, значит, размерность поверхности уровня частных интегралов не больше $[(n-1) / 2]$. Но эта размерность и не меньше, поскольку такую размерность имеет тор с образующими $\left(\exp \left(\Omega^{2 r+1} t\right) p, \exp \left(\Omega^{2 r+1} t\right) \gamma\right)$. Это означает, что каждая точка траектории искомого целого решения имеет вид $(A(t) p, A(t) \gamma)$, где $A(t) \in \operatorname{So}(n-1), \dot{A}(t)=A(t) C(t), C(t) \in \operatorname{so}(n-1),[C(t), \Omega]=0$. Кроме того, поскольку мы ищем линейное решение, то $C(t) \equiv$ const.

Подставив это представление в систему (3), получим

$$
\left\{\begin{array}{l}
A C p=\lambda \Omega A p+\mu A \gamma, \\
A C \gamma=-\Omega A \gamma-x A p .
\end{array}\right.
$$


Поскольку $[C(t), \Omega]=0$, то окончательно имеем линейную систему, в которой $C, p, \gamma$ не зависят от времени, т.е. являются по сути начальными условиями:

$$
\left\{\begin{array}{l}
C p=\lambda \Omega p+\mu \gamma \\
C \gamma=-\Omega \gamma-x p
\end{array}\right.
$$

Полученная система легко решается:

$$
\left\{\begin{array} { l } 
{ ( C - \lambda \Omega ) p = \mu \gamma , } \\
{ ( C + \Omega ) \gamma = - x p }
\end{array} \Longleftrightarrow \left\{\begin{array}{l}
(C-\lambda \Omega) p=\mu \gamma \\
(C+\Omega)(C-\lambda \Omega) p=-\mu x p .
\end{array}\right.\right.
$$

Найти собственные векторы оператора $(C+\Omega)(C-\lambda \Omega)$ достаточно просто, тем самым, задача нахождения целых решений многомерной задачи Лагранжа для интеграла $\Omega$ общего положения полностью решена. Отметим также, что необходимое начальное условие $(p, \gamma) \equiv 0$ выполнено, поскольку матрица $(C-\lambda \Omega)$ (или $(C+\Omega))$ кососимметрична.

ТеОрема 12. Все целье решения многомерного обобщения случая Лагранжа задачи о движении тяжелого твердого тела

$$
\left\{\begin{array}{l}
\dot{p}=\lambda \Omega p+\mu \gamma \\
\dot{\gamma}=-\Omega \gamma-x p \\
\dot{x}=(p, \gamma)
\end{array}\right.
$$

для кососимметрической матричы общего положения $\Omega \in \operatorname{so}(n-1)$ имеют вид

$$
\left\{\begin{array}{l}
p(t)=\exp (C t) p_{0} \\
\gamma(t)=\exp (C t) \gamma_{0} \\
x(t)=x_{0}
\end{array}\right.
$$

где $[C, \Omega]=0, C \in \mathrm{so}(n-1), p_{0}-$ собственный вектор оператора $(C-\lambda \Omega)(C+\Omega)$; $-\mu x_{0}$ - его собственное значение; $\gamma_{0}$ - произвольное начальное значение $\gamma$.

Несложно убедиться в том, что в классическом случае $n=3$ теорема 12 дает известное целое решение. Напомним, что определяющие соотношения классического решения являются следующими:

$$
\left(\dot{\gamma_{3}}\right)^{2} \equiv 0, \quad p_{1}=\lambda \gamma_{1}, \quad p_{2}=\lambda \gamma_{2}, \quad \lambda=\text { const }
$$

а решения $\gamma_{1}(t), \gamma_{2}(t)$ выражаются через синусы и косинусы.

Дифференциальные уравнения классического случая Лагранжа имеют вид

$$
\left\{\begin{array}{l}
k \dot{p}_{1}=(k-1) p_{2} p_{3}+r_{3} \gamma_{2}, \\
k \dot{p}_{2}=(1-k) p_{1} p_{3}-r_{3} \gamma_{1}, \\
\dot{p}_{3}=0 \\
\dot{\gamma_{1}}=p_{3} \gamma_{2}-p_{2} \gamma_{3}, \\
\dot{\gamma_{2}}=p_{1} \gamma_{3}-p_{3} \gamma_{1} \\
\dot{\gamma_{3}}=p_{2} \gamma_{1}-p_{1} \gamma_{2} .
\end{array}\right.
$$


Чтобы перейти от системы (12) к системе (13), надо сделать следующие переобозначения:

$$
\begin{gathered}
\Omega \rightarrow\left(\begin{array}{cc}
0 & -p_{3} \\
p_{3} & 0
\end{array}\right), \quad p \rightarrow\left(\begin{array}{c}
p_{2} \\
-p_{1}
\end{array}\right), \quad \gamma \rightarrow\left(\begin{array}{c}
\gamma_{1} \\
\gamma_{2}
\end{array}\right), \\
x \rightarrow \gamma_{3}, \quad \lambda \rightarrow \frac{1-k}{k}, \quad \mu \rightarrow-\frac{r_{3}}{k} .
\end{gathered}
$$

Теперь мы видим, что в силу теоремы $12\left(\dot{\gamma}_{3}\right)^{2} \equiv 0$, следовательно, в силу (13) $p_{2} \gamma_{1}-p_{1} \gamma_{2} \equiv 0$ или $p_{1}=\lambda \gamma_{1}, p_{2}=\lambda \gamma_{2}$. Наконец, в силу линейности решения системы (13) последние два соотношения выполняются при фиксированном $\lambda$.

\section{Список литературы}

[1] С.В. Манаков, "Замечание об интегрировании уравнений Эйлера динамики n-мерного твердого тела", Функи. анализ и его прил., 10:4 (1976), 93-94; англ. пер.: S. V. Manakov, "Note on the integration of Euler's equations of the dynamics of an $n$-dimensional rigid body", Funct. Anal. Appl., 10:4 (1976), 328-329.

[2] А. С. Мищенко, А. Т. Фоменко, "Уравнения Эйлера на конечномерных группах Ли", Изв. АН СССР. Сер. матем., 42:2 (1978), 396-415; англ. пер.: А. S. Miščenko, A. T. Fomenko, "Euler equations on finite-dimensional Lie groups", Math. USSR-Izv., 12:2 (1978), 371-389.

[3] M. Adler, P. van Moerbeke, "Completely integrable systems, Euclidean Lie algebras, and curves", Adv. in Math., 38:3 (1980), 267-317.

[4] M. Adler, P. van Moerbeke, "Linearization of Hamiltonian systems, Jacobi varieties and representation theory", Adv. in Math., 38:3 (1980), 318-379.

[5] T. Ratiu, P. van Moerbeke, "The Lagrange rigid body motion", Ann. Inst. Fourier (Grenoble), 32:1 (1982), 211-234.

[6] Ю. А. Архангельский, Аналитическая динамика твердого тела, Наука, М., 1977.

[7] А.В. Борисов, И. С. Мамаев, Современные методы теории интегрируемых систем, Ин-т компьютерных исследований, М.-Ижевск, 2003.

[8] V. Dragović, B. Gajić, "An $L-A$ pair for the Hess-Apel'rot system and a new integrable case for the Euler-Poisson equations on so(4) $\times$ so(4)", Proc. Roy. Soc. Edinburgh Sect. A, 131:4 (2001), 845-855.

[9] Б. А. Дубровин, В.Б. Матвеев, С.П. Новиков, "Нелинейные уравнения типа Кортевега-де Фриза, конечнозонные линейные операторы и абелевы многообразия", УМН, 31:1 (1976), 55-136; англ. пер.: В. A. Dubrovin, V. B. Matveev, S.P. Novikov, "Non-linear equations of Korteweg-de Vries type, finite-zone linear operators, and Abelian varieties", Russian Math. Surveys, 31:1 (1976), 59-146.

[10] А. В. Беляев, "О движении многомерного тела с закрепленной точкой в поле силы тяжести", Матем. сб., 114(156):3 (1981), 465-470; англ. пер.: A. V. Beljaev, "On the motion of a multidimensional body with fixed point in a gravitational field", Math. USSR-Sb., 42:3 (1982), 413-418.

[11] A. I. Bobenko, A. G. Reyman, M. A. Semenov-Tian-Shansky, "The Kowalewski top 99 years later: A Lax pair, generalizations and explicit solutions", Comm. Math. Phys., 122:2 (1989), 321-354.

[12] A. Belyaev, "The factorization of the flow defined by the Euler-Poisson equations", Methods Funct. Anal. Topology, 7:4 (2001), 18-30. 
[13] А.В. Беляев, "О целых решениях уравнения Эйлера-Пуассона", Укр. матем. журн., 56:5 (2004), 677-686; англ. пер.: A. V. Belyaev, "Entire solutions of the Euler-Poisson equations", Ukrainian Math. J., 56:5 (2004), 817-829.

[14] В.И. Арнольд, Математические методъ классической механики, Наука, М., 1989; англ. пер.: V. I. Arnold, Mathematical methods of classical mechanics, Grad. Texts in Math., 60, Springer-Verlag, New York, 1989.

[15] А.А. Кириллов, Элементы теории представлений, Наука, М., 1972; англ. пер.: А. A. Kirillov, Elements of the theory of representations, Springer-Verlag, Berlin-Heidelberg-New York, 1976.

[16] J. Marsden, A. Weinstein, "Reduction of symplectic manifolds with symmetry", Rep. Mathematical Phys., 5:1 (1974), 121-130.

[17] А.В. Бочаров, А. М. Виноградов, "Гамильтонова форма механики с трением, неголономной механики, инвариантной механики, теории преломления и удара", добавление II к статье: А. М. Виноградов, Б.А. Купершмидт, Структура гамильтоновой механики, УМH, 32:4 (1977), 228-234; англ. пер.: А. V. Bocharov, A. M. Vinogradov, "The Hamiltonian form of mechanics with friction, non-holonomic mechanics, invariant mechanics, the theory of refraction and impact", appendix II: A. M. Vinogradov, B.A. Kupershmidt, The structures of Hamiltonian mechanics, Russian Math. Surveys, 32:4 (1977), 235-241.

[18] А. С. Мищенко, "Интегралы геодезических потоков на группах Ли", Функи. анализ и его прил., 4:3 (1970), 73-77; англ. пер.: А. S. Mishchenko, "Integral geodesics of a flow on Lie groups", Funct. Anal. Appl., 4:3 (1970), 232-235.

[19] T. Ratiu, "Euler-Poisson equations on Lie algebras and the $N$-dimensional heavy rigid body", Amer. J. Math., 104:2 (1982), 409-448.

[20] А. С. Мищенко, А. Т. Фоменко, “Обобщенный метод Лиувилля интегрирования гамильтоновых систем", Функи. анализ и его прил., 12:2 (1978), 46-56; англ. пер.: A. S. Mishchenko, A. T. Fomenko, "Generalized Liouville method of integration of Hamiltonian systems", Funct. Anal. Appl., 12:2 (1978), 113-121.

[21] M. Rais, "L'indice des produits semi-directs $E \times_{\rho} g . ", C$. R. Acad. Sci. Paris Sér. A-B, 287:4 (1978), 195-197.

[22] А.В. Болсинов, "Согласованные скобки Пуассона на алгебрах Ли и полнота семейств функций в инволюции", Изв. АН СССР. Сер. матем., 55:1 (1991), 68-92; англ. пер.: A. V. Bolsinov, "Compatible Poisson brackets on lie algebras and completeness of families of functions in involution", Math. USSR-Izv., 38:1 (1992), 69-90.

[23] А. В. Болсинов, А. В. Борисов, "Согласованные скобки Пуассона на алгебрах Ли", Матем. заметки, 72:1 (2002), 11-34; англ. пер.: A. V. Bolsinov, A. V. Borisov, "Compatible Poisson Brackets on Lie Algebras", Math. Notes, 72:1 (2002), 10-30.

\section{А. В. Беляев (А. V. Belyaev)}

Донецкий государственный университет управления, Украина

E-mail: nika@vnet.dn.ua
Поступила в редакцию 05.05.2010 и 11.05 .2011 